\title{
Rethinking the Roles of Actors in the Mobility of Healthcare Services
}

\author{
Tiko Iyamu ${ }^{1}$, Suama Hamunyela ${ }^{2}$, and Sharol Sibongile Mkhomazi ${ }^{3}$ \\ ${ }^{1}$ Polytechnic of Namibia, Department of Business Computing, Windhoek, Namibia \\ connectvilla@gmail.com \\ ${ }^{2}$ Namibia University of Science and Technology, \\ Department of Informatics, Windhoek, Namibia \\ slhamunyela@polytechnic.edu.na \\ ${ }^{3}$ Tshwane University of Technology, Department of Office Management and Technology, \\ Pretoria, South Africa \\ mkhomaziss@tut.ac.za
}

\begin{abstract}
Patients seek attention and treatments to various types of diseases and symptoms. Diseases infection and symptoms are often not predictive. Normally, there is a spread and movement of people across the geographical locations, of both the rural and urban communities, in countries including Namibia. As such, healthcare could be needed at any location, and at anytime. There is significant mobility of individuals and groups within a country. Unfortunately, the healthcare services are not always as mobile at the level and speed that individuals and groups does in Namibia. Hence, there is need for the mobility of healthcare services at both primary and secondary healthcare levels, particularly in the developing countries, such as Namibia.

The population of Namibia is scantly spread among its towns and cities. The major towns and cities are situated, in the average of $175 \mathrm{~km}$ far apart from each other, in the country's $825,418 \mathrm{~km}$ square landscape. The spread necessitates movements of individuals and groups, particularly the old, poor, and nomadic people. Unfortunately, healthcare records in the country are not centralised and virtualised, making accessibility into patients' records difficult or impossible, from any location. As a result, healthcare service delivering is challenged. This study therefore explored and examined the possibility of mobility of healthcare services to those who live in the country.

The study employed the qualitative research method, within which data was gathered from primary healthcare service providers, using open-ended questionnaires. The Moments of Translation from the perspective of actor network theory (ANT) was used as a lens in the analysis of the data, to examine and understand the power and factors, which influences mobility of healthcare service in Namibia. Categorisation of Patients, Response Time, Understanding the Actors, Actors' participatory to service delivery, and Actors' Alliance were found to be the influencing factors in the provision of mobility of healthcare services.
\end{abstract}

Keywords: Healthcare services, Actor Network Theory. 


\section{Introduction}

The movement and spread of the population in developing countries is argued to impact healthcare service provision [1]. To ensure effective healthcare services provision, states and healthcare organisations are engaged in transforming the industry. According to [2], to improve the quality and efficiency of healthcare many hospitals are involved in extensive efforts to substitute electronic patient records for paper records. Another effort that has been made by some organisations is the integration of health information systems to improve quality of healthcare service.

Also, the shift from curative to planning and preventing of disease outbreaks and control has significantly necessitated the need for healthcare data management, efficient service delivery, healthcare information flows between health practitioners and patients, as well as information sharing between healthcare levels of operandi [3]. The mission of curative, preventing and disease control can only be made possible if the information of the whole population based is made available to policy makers, healthcare profession, administrators, donors and all healthcare organisations.

However, different categories of patients exist in the healthcare sector and the needs for healthcare services are diverse. There is the nomadic patient. This inflates the need to investigate different dimensions of healthcare service provisions processes in a country. In [4] argument, there is a scenario where the patient may visit a different healthcare organisation, either because the patient is dissatisfied with the treatment of his or her previous visit or the patient moves to a different location. Distinctively in this case is the mobility of healthcare services in Namibia.

Mobility in this paper refers to the state of easy accessibility of health services from any geographical location. The essentiality of mobility of healthcare is centred on factors such as portability, transferability and availability of healthcare information including real-time interaction between healthcare providers and the needing [5]. In healthcare, mobility is typically associated with mobile healthcare systems and applications, the use of health public kiosk, cellular phone devices, and other portable computing devices [6]. This paper argues that mobility can also be classified by the availability of healthcare services at different levels of healthcare operandi.

Mobility of healthcare services could be translated by various human actors (patients and healthcare workers), based on the different moments. Translation is a key tenet of actor network theory [7]. In actor network theory (ANT), translation is influenced by interest of the actors [8]. Translation takes place between the object and the actors it encounters as the initial program or script is altered through interaction.

ANT is popular for its ability to provide a rich and dynamic way of bringing together the socio-technical and non-technical aspects of the organization [9]. In ANT, society and organisations are a formation of different agents, and the agents interact to form heterogeneous networks [10]; [11], [12]. Networks define, describe and provide substance to agents. ANT then, deeply question and provide retorts to the existence of strong and weak (thus power) networks.

The Namibian healthcare levels of operandi cover both rural and urban areas following the thirteen political and administrative regional demarcations of the country. As a developing country, majority of Namibians still resides in rural areas. There is a significance movement of people between urban and rural areas. 
The remainder of the paper in divided into five main sections. The first and second sections cover the literature review and research methodology, respectively. The third section presents the data analysis, which the findings. Based on the findings, the implications for the mobility of healthcare services in Namibia context are discussed in the fourth section. Finally, a conclusion is drawn.

The research was guided by two main questions: (i) what are the factors which influence the mobility of healthcare services in Namibia? (ii) What are the roles of human and non-human actors in the mobility of the mobility of healthcare services?

\section{Healthcare Service}

The healthcare sector in many countries consists of a large contingence of institutions and organisations, ranging from centres, small and medium to large and technologically advanced hospitals [13]. The institutions are classified differently in many countries. In Namibia for example, the healthcare centres are classified according to geographical areas, namely, community (constituency) and district level, regional level and national level [14]. The levels of categories cover both rural and urban geographical locations. As in many developing countries, in Namibia, $58 \%$ of the population resides in rural areas while the remaining percentage resides in urban areas [15].

Due to the essentiality of healthcare, different studies have been conducted in pursuit to establish and describe health services to different healthcare seekers. [16] examined how mobile health could be used as an innovative solution, to providing healthcare services to patients who reside in remote locations. The later stated that locality influences healthcare service delivery, therefore Mobile healthcare services are essential. This is affirmed by [5] stating that, Mobile health greatly benefits patients who reside in remote areas.

Due to the geographical spread of citizens, mobile healthcare is highly essential, however, challenges do exist. According to [16], healthcare providers in rural areas face numerous challenges in providing coherent and integrated services as compared to those in urban areas, which results from lack of mobile healthcare facilities. Also, the approaches that are often used by primary healthcare service providers in the rural areas are primitive and obsolete [17]. This also could be attributed to the fact the recipients of the services do question or protest the act by the service providers.

[5] emphasised that mobility means the use of mobile technologies to access healthcare service, from different places, at the same time; different places, at the different times; different time, different places; and same time, same place. Mobile of healthcare services includes electronic records, shared and accessed via mobile devices among healthcare providers and receivers. It is assumed that, with the mobile health technologies, healthcare problems, such as inadequacy of doctors and poor clinical examination as a result of insufficient skilled workers and scarcity of centre are eradicated [18]. Since available healthcare providers can now remotely interact with patients and conduct analysis remotely. According to [19], M-health offers a variety of benefits, such as timely access, monitoring patients remotely, and improved quality of patients care, to healthcare organisation, providers and patients. 
The need for accessibility and quality of health information particularly in developing countries such as Namibia necessitates refection and attention. Thus, there is need for investigation on the mobility of services to improve delivering of healthcare. It has been empirically unveiled, it is important to have healthcare services at the communities' disposal, and at any location, as opposed to the difficulty of accessibility due to distances. The investigation to ensure achieving this objective was carried through a study. The methodology that was applied in the study is explained next.

\section{Methodology}

The case study research approach and the qualitative interpretive research method were employed. The case study approach was selected mainly because of its focus on real-life situation. According to [20], a case study can be described as the investigation of a contemporary phenomenon within a real-life context. The Namibian Ministry of Health and Social Services (MoHSS) was used as the case, in the study. In addition the MoHSS, the general populace were allowed to participate in the study.

Questionnaires and interviews were used, from the perspective of qualitative method. This was to allow the participants to share their subjective view of how they provide and receive healthcare services in the country. [21] argued that the qualitative method recognises and focuses on the subjective meaning which the participants bring to the context of the study. Upon completion of the questionnaire, follow up telephonic interviews were conducted to establish deeper understanding of the formation of networks and build of alliances. The two techniques are well established forms of data collection in social science studies.

The participants in the study were identified. A total of 23 people participated in the study. The participants included nurses (6), doctors (4), and patients (8) and nonpatients (5), across the country. As at the time of the study, each of the participants had been in service, in their profession for at least five years. This was to ensure the quality of data obtained from them, in terms of the services that they render to nomadic patients of the country.

The data analysis was carried out, using the moments of translation from the perspective of actor network theory (ANT). ANT was selected mainly because it allows us to examine the process of change, and the interaction that take place between technical and non-technical actors. Without the use of ANT, it would have been difficult or impossible to understand and exhume the processes and actors' influences in the mobility of healthcare services in Namibia. Translation occurs when actors start to define roles, distribute and redistribute roles and power, describe a scenario to form alliances of technical and non-technical. Translation stage involves a process of change, which occur via four major moments. The ANT translation stage involves a process of change, which occur via four major moments: problematization, interessement, enrolment and mobilization. This occurs when actors start to define roles, distribute and redistribute roles and power, describe a scenario to form alliances of technical and non-technical.

The Moments of Translation elements can be summarised as follows: (i) Problematization is the stage which reveals what necessitate the network formation. [22] 
elaborated that during problematisation, actors establish themselves as an obligatory passage point (OPP) between them and the network for indispensability. (ii) Interessement: at this stage, actors consciously or unconsciously reveal their individual or groups' interest on the item that has been problematized by the focal actor. (iii) Enrolment: process begins with a primary agent imposing their will on others for actions to be executed; this act requires yielding from different actants. (iv) Mobilization: an enrolled actor speaks on behalf of the network, and tries to an interest to persuade others to partake in the activities of the network.

\subsection{Namibian Ministry of Health and Social Services (MoHSS): The Case Studied}

As it is in many countries, the Ministry of Health and Social Services (MoHSS) of Namibia coordinates and oversee the activities of the medical and health sectors in the country. The MoHSS is mandated to provide an integrated, affordable, accessible, quality health and social welfare services, which is responsive to the needs of the Namibian population [17]. The population seeks services from the available healthcare facilities which are located at different levels of operandi in the Namibia [14]. Processes and activities of the MoHSS are mostly performed manually (paperbased) or through health information systems (HIS). This can be a huge setback for the services which the MoHSS provides to the communities in the country such as distribution of the HIV treatment and immunisation against contiguous decease's outbreaks. Worse, the manual approach significantly delays centralisation of processes and activities [14]. This indeed is dangerous to patient needs, particularly those who are of intensive and chronic nature and nomadic patients.

In addition, there is mobility of individuals and groups within a country. Hence, there is need for the inclination of centralizing healthcare services at both primary and secondary healthcare levels in developing and developed countries. In Namibia, the geographical spread of the population, the widely held image of the healthiness of people in rural area, different groups with needs for healthcare (the old, the poor, nomadic people est.) necessitates the mobility of healthcare services.

\section{$4 \quad$ Data Analysis}

As mention earlier in the methodology section, the analysis of the data was done, using the lens of actor network theory. The components of ANT that were followed in the analysis include Actor-Network, and Moments of Translation. In each of the sections of the analysis, the findings are presented and discussed.

Patients are attended to when seeking for health services at the centre, where a discussion (Translation) between the patient and the healthcare takes place. It is procedural to bring along a medical passport (a form of identity which issued by healthcare service providers) when seeking for health services and routine for the healthcare provider to note in the health passport the diagnosis taken and prescriptions. Interactions between healthcare and patients can be classified as part of a durable network that exists to offer services. It is a durable network because a fixed health centre exists and there are actants stationed at each centre. 
Currently there are no records readily available when attending to in transit patients except for the health pass port and the medication labels which might not be available at times or lost. For example HIV patients who have been under the care of private doctors and wish to move to state hospitals might not be attended to or attended to after a long time due to communication break down between public and private centres. One of the common challenges encountered by the healthcare providers was a repetition of treatment course which were not effective. There were no records to show that the same treatment or test was carried out, and that it was not effective. One way to overcome these issues is implementation of an Electronic Patient Monitoring System (EPMS) and Electronic Dispensary Tool (EDT) linked to all health facilities providing HIV care for patients to be helped without relying on hard copies only.

\subsection{Actor-Network}

The human actors in the healthcare sector includes healthcare needing people, healthcare professionals (Doctors and Nurses), and the government MoHSS. The nonhuman actors consist of processes, ICT artefacts and other medical tools. As at the time of this study, different networks existed, some of them were in the categories of nurses' professionals, urban patients, rural patients, and tribal patients.

The actors had different interest. Each of the actors made a difference, either as a receiver or provider of healthcare service. The difference was based on the impact that the actors had on each other. For example, the knowledge a professional acquired in the course of providing health service to a patient.

Currently, there exist different networks to which actors belong. The enrolment of actors in the different networks was influenced by their interests. As gathered from the participants in the study, the primary interest of the general actors were, to receive service from the practitioners, and to render service to the patients. These interests were however, influenced and manifested by other factors and interests, which included spoken language, culture, and accessibility.

\section{Findings: Categorisation of Patients}

Categorisation of patients was found to be significant, due to the different healthcare services they require, and how the services could be provided and by whom. The categorisation is divided into grouping and indexing:

i. Grouping - the actors require groupings, in terms of networks. The different networks, formed in accordance to categories, such as health related challenge, gender, spoken language, and resident location. This will help in terms of referral of patients, to specialist.

ii. Index key - indexing of patients' record is critical. This is to enable searches, in the different categories, such as spoken language, tribal origin, current location, and illness type. This will help improve response time.

Some of the patients could only enrol in the network where there preferred language was used as the medium of communication. Even though interpreters were readily available, they preferred to express themselves than the use of translation. Enrolment into a network by many patients in some parts of the country was influenced by their 
culture. According to some of the participants, their tradition does not allowed them to be treated by professional of opposite sex.

\subsection{Moments of Translation}

The moments which consist of four stages, namely problematisation, interessement, enrolment and mobilisation was employed in the analysis primarily to examine and understand the interactions which happen amongst the actors. This was to further understand the suitability, and transformation of the current status quo to era of mobility of service within the healthcare environment.

\section{- Problematisation}

The activities of the healthcare sector were initiated, problematised through two primary channels, patient and the government. The patients problematise an activity through illnesses, which were often varied. While the other focal actor, the government does same, problematise its healthcare related activities through the Minister of the Ministry of Health and Social Services (MoHSS).

The patients or their representatives (guardian) report incidents of illness, including vehicle accident involving human at the nearest healthcare centre. The incidents were recorded by the front-office professional, who normally were the Nurses. The Nurses escalate the incidents to the appropriate or respective specialist for further and detailed attention. Another criterion that was used for the referral of patients was language, to enable communication and precise understanding between actors (patient and specialist). The Nurses does so, record and escalate incidents based on the power bestowed on them by the authority of the organisation, which comes from their qualification to practice.

The government problematises all initiatives relating healthcare in the country through the Minister of health. The Minister further problematises the initiatives to heads of units and departments of agencies and organisation within the health sector, through workshops and strategic meetings. Unfortunately, the initiatives are not fit for the districts and rural communities, where they are considered needed most e.g. Unavailability of medical reports 'breaks' the continuity of HIV care between health districts.

Healthcare service providers are stationed at different locations of operands. The spread of the operands are far apart, of about $150 \mathrm{kms}$ in average. The travelling and nomadic people are therefore forced to seek healthcare service from the nearest location of medical operand. There is a national policy which is intended to address the challenges of patients who are "on-transits" within the country. The policy defines "on-transit" as a maximum duration of three months stay from original or residence location. The implication is that, a patient who exceed that period may be denied the privilege which the on-transit healthcare service offers. Also, on-transit patient require a letter or medical passports in other to receive medical attention. The requirement is be able to access medical history, which includes previous diagnoses, treatments, and consultations. Unfortunately, some healthcare service providers are adamant on the requirement before service. The challenge is that many people inevitably lose or damage their medical passport. Some people do not always have the 
passport with them as the medical incidents are not always predictive. Even though the health passport is replaceable, the process is not real-time. As such, it delays treatment to the patient, worst, if the incident is severe.

\section{Findings: Response Time}

Following the process of problematization, from the moment of translation, we found Response Time to be crucial in delivering healthcare service to patients. This is even more critical because of the distances the patients have to travel to get the services. Response time is further viewed from two perspectives of significance, in the mobility of healthcare services in Namibia. The factors include the impact of bureaucracy and lack of access to real-time medical data:

$i$. Bureaucracy - the process of initiating medical attention is too bureaucratic for incidents which are health related. It is worst if the incident is severe, or become live-threatening. The situation can be more challenging in rural areas, and where there is no next of kin to provide relevant information about the patient.

ii. Real-time data - there is lack of real-time data of patients' record. This makes it difficult for healthcare providers to provide relevant and instance services to patients, or to know of previous diagnosis and prescriptions.

Our interpretation of the findings indicates that the impact of bureaucracy and lack of access to real-time medical data requires cloud computing solution, as shown in Figure 1.

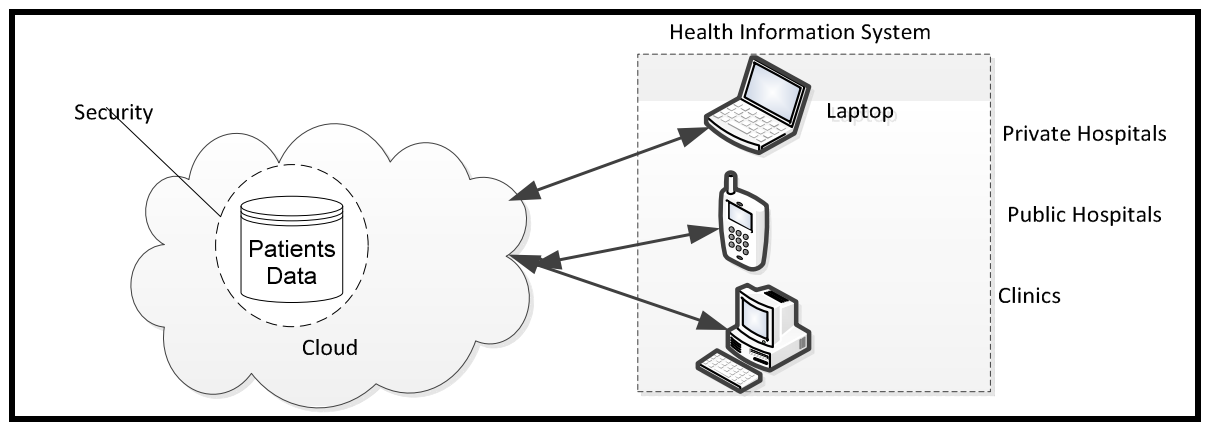

Fig. 1. Patients data in the cloud

Cloud Computing - Figure 1, depict the use of cloud computing solution to store, and access patients' data for health services in Namibia. [23] referred to cloud computing as both the applications delivered as services over the Internet and the hardware and systems software in the data centers that provide those services. This allows personnel or specialists to store and access patients' data from Private and Public hospital, including clinics, using different devices, such as laptop, mobile phones, and desktop. The architecture (Figure1), using the cloud solution, enables all healthcare medical personnel in the country to have access to real-time data for their services. 
The overall problem is the provision of healthcare services to the nomadic patients of the country. The MoHSS aim to provide effective services to all patients regardless of their dwelling status are currently a challenge, as many of the patients are given too much responsibility in addition to their individual illness. In response to the need for a more effective health services, highly qualified and skilled workforce is enrolled in this activities. Furthermore, procedures to deliver or receive healthcare services are enforced (e.g. only registered nurses can attend to patients or patients should carry their healthcare passport in case looking for healthcare services). This in turn leads to the consideration of suitable healthcare provision, healthcare received and medical history for patients. For healthcare service innovation by the MoHSS, the problematisation proposed by the instigators is that to improve healthcare services to all patients including the nomadic patients, there must be service innovations that enable the desired outcomes. This is seen as an OPP by the MoHSS to remain a competent healthcare service provider in the country. In the case of the patients, they need to be certain that they will receive adequate services at any time anywhere.

\section{- Interessemment}

As earlier revealed in the analysis, the actors had different interests. The actors were categorised into two main groups, healthcare service recipients and providers, which consist of patients and professionals, respectively. The interests of these groups were diverse, even though the primary goal was healthcare services.

The interest of the healthcare recipients were influenced by factors such as locations, type of health related service that was required, and the affordability of services. Proximity was a hindrance to many of the people who lived in the rural areas of the country. The healthcare facilities were far apart from each other. As a result, there were few options to those who need services such as Human Immunodeficiency Virus (HIV) patients, who needed to get their Antiretroviral (ARV) medications from healthcare centre. Due to the fewness of the facilities, the common ones were "General Practitioners". This made it difficult or impossible to access specialists and certain services.

The MoHSS for instance, interest concern its public mandate and social responsibility visible in different directorates and divisions that formed up the MoHSS. The MoHSS also has directorates and division managers in different roles that oversee the execution of duties (e.g. delivery of services), managing healthcare centres, recruit healthcare providers, procurement of necessary medications and other equipment's. These individuals set themselves up as the focal actors and as such, making rues for obligatory passage point.

The healthcare providers' interests were of professional progress, income, status and job descriptions. Healthcare providers in service benefited differently during the course of their duties, depending on the location and the responsibilities that they were assigned. Some of the benefits included flexibility and shifts working hours, housing subsidy, and free medical aid. Also, exposure to different health related cases which in long term can be attributed to experience gained.

ICT artefacts were used differently in the healthcare organisations to support the delivery of services, such as diagnostics and documentation of individual and group records. The use of ICT artefacts were also based on the interest of the users. 
The interests were informed by their technical skill and know-how of the technologies, and what they needed them for. Another factor was whether the technologies were supported for use in the environment that they were deployed. This played a critical factor in individual and group interest to make use of available technologies for healthcare services in Namibia. As a result, many of the professional anchor their interests on paper-based processes, which they were more comfortable with in delivering services. Paper technology is crucial to nomadic patients thus; it is required for them to forward the healthcare passport to receive the necessary services.

\section{Findings: Understanding the Actors}

In our examination of the empirical data, using the interessment tenet of the Moments of Translation, we found Understanding of the Actors to be significantly useful. In that, only through understanding of the involving actors mobility of healthcare services can be influenced. Understanding of the actors was shared from the perspective of awareness and technology know-how:

i. Awareness - the actants (healthcare providers and patients) took part in the healthcare services, including in the ways in which the services were provided and received, were based on their levels of awareness. For example some health professionals were became health professionals for personal benefits; and some patients' preference for healthcare services centres were influenced by factors, such as their knowledge of the type of services provided by the centres, the types of their illness, and knowledge of proximity to the centres.

ii. Technology know-how - healthcare providers made use of the technologies that they were familiar and comfortable with. For example, some professional could hardly use computer systems to record or access patients' data. However, different technological tools are applicable in diagnosis and other services provision

As already established, the lack of awareness and technology know-how has detrimental implication on the mobility of healthcare services. Thus, a mobile kiosk provides a redress. The use of mobile kiosk will help to reduce or eradicate factors, such as proximity, as it changes location. Also, it could assist patients to garner more information that are offered and available in the different centres, as the patients visit the kiosk in their locations.

As shown in Figure 2, the mobile kiosk is intended to continually, move from one location to another. The kiosk is expected to move within cells (geographical location covered by base station), so it could access, and be accessed by hospitals and clinics, at any location, using any mobile device. According to [24], mobiles devices include microwave ovens, Bluetooth devices, and cordless phones. However, personnel from the hospitals and clinics need to be able to make of mobile devices in order to access the mobile kiosk. 


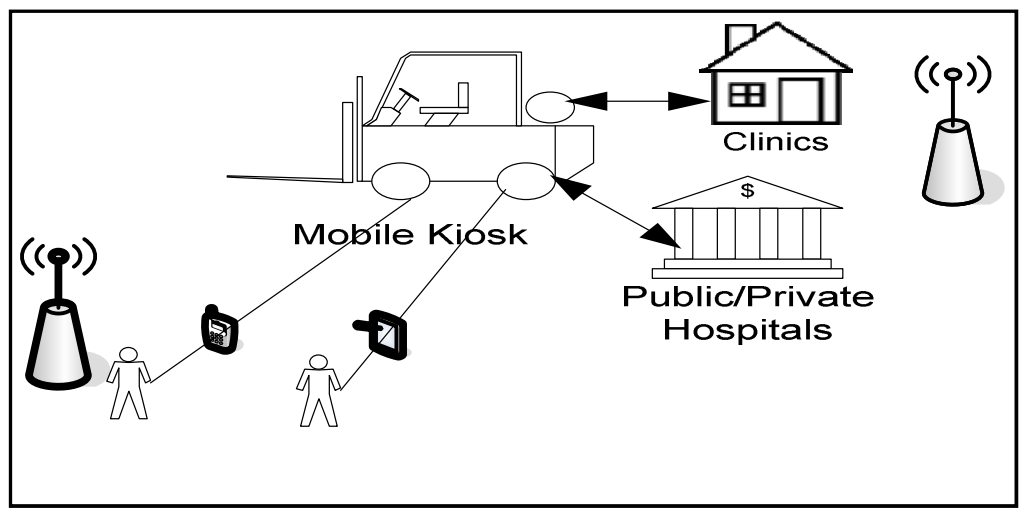

Fig. 2. Mobile Kiosk

\section{- Enrolment to the Network}

This process begins with a primary agent (in this case the MoHSS management) imposing their will on others in attempt to achieve for common goal. This was mainly because of the significant of participation of all key stakeholders, which determines the success of failure of healthcare initiatives in Namibia. However, participation was always a challenge, as a result, definition of roles and responsibilities were seen as critical.

The ministry defines the regulations, roles and responsibilities to healthcare providers, and standards of service deliveries at public healthcare centres. This process centred on negotiation and alignment of actants' interests within the different networks. The process was challenging in that it was always lengthy and difficult, and so many conflicts arose. The challenges were manifestation of the different cultural background, interests' focuses, as well as recording keeping. For example, some patients had relocated, and others had lost their health passport, and they needed medical attention. In such cases provision was made to create a new health passport for them through negotiation.

Even though health services were considered essential and critical, some patients would not enrol or reluctantly enrolled in the health related programs. This challenge was prevalence in the rural parts of the country. Many of the communities' members cited the same reason for their lack of participation in the health programs. Some of the reasons included accessibility, literacy, and affordability. For example, some of the participants in this study claimed that many needing healthcare are not literate enough to understand the messages which were used as medium of communication to them. Inscription occurs in this case in the sense that the primary interest of the MoHSS at different healthcare centres was to provide efficient services to all patients including the nomadic patients. Interest of all actants has been aligned and now there is a need to maintain the network. This results into a durable or not durable network.

Existence of conscious and unconscious networks of people, and their enrolments which were created overtime and space across the country signifies that there is need for durability and mobility of healthcare services. Thus, patients communicate their healthcare complains to healthcare providers, provide the healthcare passport if in possession, 
healthcare providers carry out the diagnosis process and offer prescriptions, again healthcare providers communicates the different cases encountered at reporting intervals to supervisors which in return reports to management (regional, district or national). Or though healthcare providers have power in durability of the network, MoHSS management have the most power to ensure the existence and the growth of the network.

\section{Findings: Actors' Participatory to Service Delivery}

The analysis revealed that actors' participatory, drawing from the connection and relationship between human actors and interactive systems, is essential to the mobility of healthcare services in the Namibian context. The connection and relationship between the actors is enacted by different tools and devices, to enable and encourage participation in the MoHSS initiatives on health programs, across the country. The components of the participatory factor, connectivity of the actors and interactive system:

i. Connectivity of actors - connectivity will foster actors' enrolment in the mobility of healthcare services. As shown in Figure 2, users (healthcare service providers and patients) need to have application on their devices, which will enable them to easily access the mobile kiosks. The application should be symmetrical, in that it is user friendly to both literate and illiterate actors in their needs to access the kiosks. The application will enable users to easily access the kiosks, from any location including at impromptu times.

ii. Interactive system - the interactive system establishes a step-by-step conversation, with the caller. The system is intended to scrutinize calls from the communities, thereby narrow the request or inquiring towards a specific need of the caller. This is purposely for efficiency and effectiveness of the mobile kiosks, in response to the community needs.

\section{- Mobilisation}

Due to the essentiality of health services in the country, it was vital for some actors from both MoHSS and the community to mobilise the healthcare service providers and recipients towards a common goal.

During mobilisation, some actors were assigned the role of new initiators by becoming delegates or spokesperson for the focal actor [25]. In this case, hospital matrons, and supervisors at different healthcare centres tried to mobilise healthcare providers to actively participate in the network (carry out their duties). This resulted to a stability of the process of delivering healthcare services in some areas, particularly in the urban areas.

As a result of the stability through adequate services, more patients became spokespersons to different healthcare centres. The self appointed spokespersons encouraged others with the same or different health issue to seek healthcare services from designated locations of their preference. Also, the altitude and tribal inclination were the others factors which influenced mobilisation and of patients. For example, some patients recommended others to visit or seek assistance from health centres where majority of the health professional were on their tribal origin and spoke the same language as them. 


\section{Findings: Actors' Alliance}

The actors formed formal and informal alliances. The alliances were significantly helpful in understanding the challenges factors in the quest to provide healthcare services to the various communities in the country. The alliances were seen as forms of collaboration and political affiliation:

i. Collaboration -mobility of healthcare services require collaboration between community members and healthcare providers. The collaboration could be driven by an agent, but it is the responsibility of all that are involved in the mobility of healthcare services. The collaboration could happen at different levels, such as community members, health professional, and the government (MoHSS).

Political affiliation - the mobility of healthcare services in Namibia is endangered by tribal preference for services. This manifests from political affiliation of individuals and groups. Political inclination has potentials of influencing mobilisation of actors, to participate in the mobility of healthcare service in their communities. Political inclination is critical as some actors are heterogeneous in the networks, as shown in Figure 3.

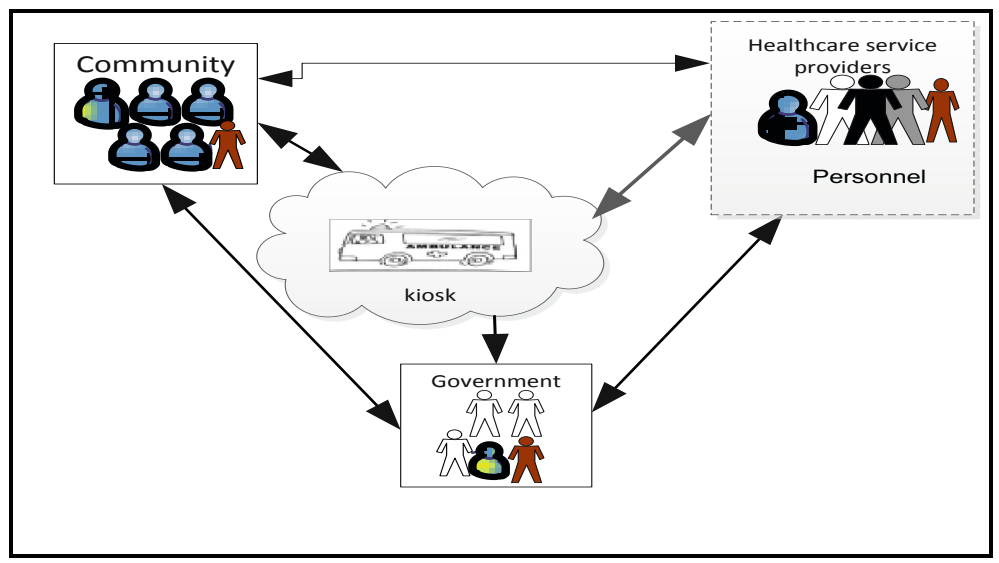

Fig. 3. Collaboration

Social Networking - the social networking approach will help to bring all actors together, in fostering the mobility of healthcare services in Namibia. According to [26], ssocial networking is a type of virtual community that has grown tremendously in popularity over the past few years. Social networking has no boundaries, in terms of spoken language, and tribal origin. It brings people of common interest together in the same network.

Another important factor of social networking is that, an actor can be heterogeneous in that the actor can belong to more than one network, as shown on figure 3 . In this instance, the actor is able to influence and connect the networks to each other. This help to eradicate or silence tribal or political inclination which has potential to hamper the common goal, of the mobility of healthcare services. 


\section{$5 \quad$ Mobility of Healthcare Services in Namibia}

People, technology and procedures are the actors in the process of healthcare service delivery. ANT's translation process that was followed in this study defines the formation of networks, which helped to identify the groupings and how they were created. The process starts with the main actor who defines the roles through problemitisation of the issue or item. This was done by localization of the issue, so as to foster relationship, and get more and regional actors to be interested, and partake in the initiative.

Although the employment of technical actants can be viewed as the consequences of the action of all actants to an extent, the ministry of health in this case has power to hire healthcare providers and extend the provision of services to all health service level of operation. This signifies authority to define procedures of service delivery e.g. change the national policy of the in transit patients or implement an Electronic Patient Management System (EPMS) at health centers. By so doing, associations of technical and non technical actants are strengthen.

Moreover, the mobility of healthcare services is enabled and constrained within network such as community by the actions of actors, which often based on individual or group knowledge. Mobilisation is necessary to educate and inform actants about the services. Patients need to know about their rights to health services at any location and the importance of a health passport.

A health passport, referral letter or medication container was issued to patients by medical personnel at different locations or health centres across the country. Thus, the patients present whenever they needed health related treatment anywhere in the country. Challenge of presenting the above is that they are vulnerable to loss or results to unclear information on the passport or letter. The consequence of such loss and unclear information make services complex and complicated to both healthcare service providers and the patients. Hence it is critical to enforce electronic systems, which allow access to patients' health related records from anywhere across the country, and on real-time.

\section{Conclusion}

This paper presents a critical analysis of the role and account of actors in the mobility of healthcare services in Namibia. The analysis is of vital important to the Namibia, and other countries which has similar setup and challenges, in that it unveiled issues that the State government and many healthcare professional are not aware of, take for granted.

In the past, and continuous (before this study), the state Government as in many developing countries continue to invest and focus on technical issues, which often become "white elephants" due to lack of usefulness. This study revealed fundamental and primary issues, which makes technology useful and ease of use in the mobility of healthcare services across Namibia.

The paper emphasises on the importance of relationships between the actors (health professional and patients), and as well the significance of networks in the mobility of healthcare services at different health operandis. As at the time of the study, patients in 
transit were handled in accordance to the national health policy as promulgated by the MoHSS. The study revealed how healthcare service providers, through the use of spoken language and tribal origin, enable, and at the same time, constrain the services that they are supposed to render to the patients for better healthcare. These factors were often conscious, and sometimes unconsciously exhibited by the actors. However, little or nothing was known of the impact, the service providers of healthcare in Namibia.

\section{References}

1. Rygh, E.M., Hjortdahl, P.H.: Continuous and integrated health care services in rural areas. A literature study. The International Electronic Journal of Rural and Remote Health Research, Education Practice and Policy 7(766), 1-10 (2007)

2. Sander-Granlien, M., Hertzum, M.: Confirmatory factor analysis of service quality dimensions within mobile telephony industry in Ghana. The Electronic Journal Information Systems Evaluation 15(2), 197-227 (2007)

3. Chaulagai, C.N., Moyo, C.M., Koot, J., Moyo, H.B.: Design and implementation of a health management in Malawi: issues inovation and results, pp. 2-10. Published by Oxford University Press in association with The London School of Hygiene and Tropical Medicine (2005)

4. Chang, P.: Modeling the Management of Electronic Health Records in Healthcare Information Systems. In: 2011 International Conference on Cyber-Enabled Distributed Computing and Knowledge Discovery (2011)

5. Fardoun, H., Cipres, A., Alghazzawi, D., Oadah, M.: KAU e-Health Mobile System. In: 13th International Congress on Human Computer Intercation. ACM (2012)

6. Cisco.: Mobility solution for healthcare: voice, text, images and information, delivered to the point of care (2007),

http://www.cisco.com/web/strategy/docs/healthcare/

07Cs1084-MobForHC_062708.pdf

7. Latour, B.: Technology is society made durable. In: Law, J. (ed.) A Sociology of Monsters: Essays on Power, Technology and Domination, pp. 103-131. Routledge, London (1991)

8. Iyamu, T.: Underpinning Theories: Order-of-Use in Information Systems Research. Journal of Systems and Information Technology 15(3), 1-13 (2013)

9. Wickramasinghe, N., Bali, R., Goldberg, S.: Using S'ANT for facilitating superior understanding of key factors in the design of a chronic disease self-management model. Actor Network Theory and Technology Innovation: Advancements and New Concepts Journal, Information Science Reference, Hershey, NY (2011)

10. Law, J.: Notes on the theory of the Actor Network: ordering, strategy and heterogeneity (1992), http: / / comp. lancs.ac.uk/sociology/soc054jl.html

11. Tatnall, A., Gilding, A.: Actor-Network theory and Information Systems Research. In: Proceeding of the 10th Australian Conference on Information Systems (ACIS), Wellington, Victoria University of Wellington (1999)

12. Creswell, K., Worth, A., Sheikh, A.: Actor-Network theory and its role in understanding the implementation of information technology developments in healthcare. BMC Medical Informatics and Decision Making, 10-67 (2010)

13. Braa, J., Hanseth, O., Heywood, A., Mohammed, W., Shawn, B.: Developing information systems in developing country: The flexible standards strategy. MIS Quartely (31), 1-22 (2007) 
14. Hamunyela, S., Iyamu, T.: Readness Assessment model for the deployment of health information systems in the Namibian MoH. In: International Federation for Information Processing, 12th Internation Conference on Social Implications of Computer in Developing Countries, Jamaica (2013)

15. NPC: Namibia 2011 population and housing census (2012), http: / / www.npc.gov.na

16. Rygh, E.M., Hjortdahl, P.: Continuous and integrated health care services in rural areas: a literature study. Rural and Remote Health 7, 766 (2007)

17. MoHSS.: Integrated healthcare delivery the challenge and implementations (2012), http: / / www. healthnet.org.na/documents.html

18. Narang, J.K.: Quality of Healthcare Services in Rural India: The User Perspective. VIKALPA, 51-60 (2011)

19. Istepanian, R.J.: Guest Editorial Introduction to the Specialon M-Health: Beyond Seamless Mobility and Global Wireless Health-Care Connectivity. IEEE Transactions on Information Technology in Biomedicine, 405-414 (2004)

20. Yin, R.K.: Case Study Research Design and Methods, 3rd edn. Sage Publications, UK (2003)

21. Hovorka, D.S., Lee, A.S.: Reframing Interpretivism and Positivism as Understanding and Explanation: Consequences for Information Systems Research. In: International Conference on Information Systems, Paper 188 (2010)

22. Uden, L., Francis, J.: Service Innovation using Actor Network theory. Actor Network Theory and Technology Innovation: Advancements and New Concepts Journal, Information Science Reference, Hershey, NY (2011)

23. Armbrust, M., et al.: A view of Cloud Computing. Communications of the ACM 53(4), 50-58 (2010)

24. Haeberlen, A.: Practical Robust Localization over Large-Scale 802.11 Wireless Networks. In: Proceedings of the 10th Annual International Conference on Mobile Computing and Networking (2004)

25. Iyamu, T., Tatnull, A.: The impact of netwrork of actors on the infomation technology. Actor Network Theory and Technology Innovation: Advancements and New Concepts Journal, Information Science Reference, Hershey, NY (2011)

26. Dwyer, C., Hiltz, S., Passerini, K.: Trust and privacy concern within social networking sites: A comparison of Facebook and MySpace. In: The Proceedings of the Thirteenth Americas Conference on Information Systems (2007) 